\title{
Could KL-6 levels in COVID-19 help to predict lung disease?
}

\author{
AN Frix ${ }^{1 *}$, L. Schoneveld ${ }^{2}$, A. Ladang ${ }^{2}$, M. Henket ${ }^{1}$, B. Duysinx ${ }^{1}$ F. Vaillant ${ }^{1}$, B. Misset ${ }^{3}$, M. Moutschen ${ }^{4}$, R. Louis ${ }^{1}$, \\ E. Cavalier ${ }^{2+}$ and J. Guiot ${ }^{1+}$
}

\begin{abstract}
Background: Coronavirus disease COVID-19 has become a public health emergency of international concern. Together with the quest for an effective treatment, the question of the post-infectious evolution of affected patients in healing process remains uncertain. Krebs von den Lungen $6(\mathrm{KL}-6)$ is a high molecular weight mucin-like glycoprotein produced by type II pneumocytes and bronchial epithelial cells. Its production is raised during epithelial lesions and cellular regeneration. In COVID-19 infection, KL-6 serum levels could therefore be of interest for diagnosis, prognosis and therapeutic response evaluation.
\end{abstract}

Materials and methods: Our study retrospectively compared KL-6 levels between a cohort of 83 COVID-19 infected patients and two other groups: healthy subjects $(n=70)$ on one hand, and a heterogenous group of patients suffering from interstitial lung diseases ( $n=31$; composed of 16 IPF, 4 sarcoidosis, 11 others) on the other hand. Demographical, clinical and laboratory indexes were collected. Our study aims to compare KL-6 levels between a COVID-19 population and healthy subjects or patients suffering from interstitial lung diseases (ILDs). Ultimately, we ought to determine whether KL-6 could be a marker of disease severity and bad prognosis.

Results: Our results showed that serum KL-6 levels in COVID-19 patients were increased compared to healthy subjects, but to a lesser extent than in patients suffering from ILD. Increased levels of KL-6 in COVID-19 patients were associated with a more severe lung disease.

Discussion and conclusion: Our results suggest that KL-6 could be a good biomarker to assess ILD severity in COVID-19 infection. Concerning the therapeutic response prediction, more studies are necessary.

Keywords: COVID-19, Interstitial lung disease, Lung infection, Biomarker, KL-6

\section{Introduction}

The rapid outbreak of coronavirus disease 2019 (COVID19) caused by severe acute respiratory syndrome coronavirus 2 (SARS-COV-2) infection, has plainly become a public health emergency of international concern $[1$, 2]. Despite a wide range of clinical presentation and varying degrees of severity, more than 887.814 people

\footnotetext{
*Correspondence: an.frix@chuliege.be

${ }^{\dagger}$ E. Cavalier and J. Guiot contributed equally.

1 Department of Respiratory Medicine, CHU Liège, University Hospital of Liège, Domaine Universitaire du Sart-Tilman, B35, 4000 Liège, Belgium Full list of author information is available at the end of the article
}

worldwide have now recovered from this viral lung infection. Though the number of recoveries does provide solace, there is still little information about how these patients and their lung integrity will evolve throughout the post-infectious healing process.

Interstitial lung damage induced by viral infection conventionally leads to respiratory symptomatology associating cough, chest pain and dyspnea. Dyspnea may in some cases persist for several weeks due to lung parenchymal sequelae. Coronavirus are known for their potential to induce lung fibrosis [3].

In this context, it therefore appears imperative to identify potential biomarkers associated with alveolar original author(s) and the source, provide a link to the Creative Commons licence, and indicate if changes were made. The images or other third party material in this article are included in the article's Creative Commons licence, unless indicated otherwise in a credit line to the material. If material is not included in the article's Creative Commons licence and your intended use is not permitted by statutory regulation or exceeds the permitted use, you will need to obtain permission directly from the copyright holder. To view a copy of this licence, visit http://creativecommons.org/licenses/by/4.0/. The Creative Commons Public Domain Dedication waiver (http://creativeco mmons.org/publicdomain/zero/1.0/) applies to the data made available in this article, unless otherwise stated in a credit line to the data. 
pathology that could be of prognostic interest regarding the severity of lung sequelae.

Different studies highlight the importance of the quest for diagnostic or prognostic biomarkers in pulmonary fibrosing process [4-6]. Krebs von den Lungen 6 (KL-6) is a high molecular weight mucin-like glycoprotein produced by type II pneumocytes and bronchial epithelial cells. Its production is raised during epithelial lesions and cellular regeneration. In normal lungs, this glycoprotein is involved in fibroblast stimulation and apoptosis inhibition. In case of epithelial lesions, alveolo-capillary leak can occur and lead to an increase in serum KL-6 levels. Indeed, this modulation is not specific to COVID-19 infection and can be found in numerous other diseases associated with alveolar epithelial cell lesions (autoimmune diseases, radiation-associated pneumonia, drugassociated pneumonia, etc.). In COVID-19 infection, KL-6 serum levels could therefore be of interest for diagnosis, prognosis and therapeutic response evaluation.

Our study aims to compare KL-6 levels between a COVID-19 population and healthy subjects or patients suffering from interstitial lung diseases (ILDs). Ultimately, we ought to determine whether KL-6 could be a marker of disease severity and bad prognosis.

\section{Materials and methods}

Our study retrospectively compared KL-6 levels between a cohort of 83 infected patients (COVID-19 PCR positive patients hospitalized in Liège University Hospital between March 1st to April 20th 2020) and two other groups: healthy subjects $(n=70)$ on one hand, and a heterogenous group of patients suffering from ILD $(n=31$; composed of 16 IPF, 4 sarcoidosis, 11 others) on the other hand.
Demographical (including age, sex, past medical history), clinical (including oxygen levels, Intensive Care Unit (ICU) indication) and admission laboratory indexes (including serum CRP, serum IL-6/KL-6, serum LDH, complete blood count, renal and liver functions) were collected. Chemistry analyses were run on the Abbott Alinity platform (Abbott Park, IL, USA) and KL-6 were measured with the Fujirebio Lumipulse 1200 instrument (Tokyo, Japan). High levels of KL-6 (45.6 UI/L) where defined as the value above the mean of the normal population +2 SD $(260.42 \mathrm{UI} / \mathrm{L}+(2 \times 95.3))$.

\section{Results}

Baseline characteristics and comparison between the three groups (Healthy Subjects, COVID-19 patients, ILD patients) are presented in Table 1.

Our results (Fig. 1) showed that KL-6 levels in COVID-19 patients were increased compared to healthy subjects, but to a lesser extent than in patients suffering from ILD (Fig. 1a). Of interest, increased levels of KL-6 in COVID-19 patients were associated with a more severe lung disease based on oxygen levels at admission to the ambient air [median $\mathrm{SpO}_{2}$ of $90 \%$ in high KL-6 level patients $(\mathrm{n}=36)$ versus $94 \%$ in low KL-6 level patients $(n=47)] ; p=0.013 ; r=-0.271$, Fig. $1 b$, e). However, high KL-6 were not linked to severe dyspnea $(p=0.585)$, or to ICU admission $(p=0.434)$. Similarly there was no association between high KL-6 levels and mortality $(p>0.05)$. Concerning laboratory values, despite an increase in CRP and fibrinogen levels in COVID-19 patients, there was no correlation between high KL6 levels and CRP $(\mathrm{p}=0.482)$ or fibrinogen $(\mathrm{p}=0.288)$. Confirmatory to previous results focusing on biological markers associated with severe COVID19 infection, high KL-6 was correlated with high LDH

Table 1 Baseline characteristics of the 3 groups, and comparison of their respective features

\begin{tabular}{|c|c|c|c|c|c|c|}
\hline \multicolumn{4}{|c|}{ Baseline characteristics } & \multicolumn{3}{|l|}{$P$ values } \\
\hline & $\mathrm{HS}^{\mathrm{a}}(\mathrm{N}=70)$ & COVID19 $(\mathrm{N}=83)$ & $\operatorname{ILD}(\mathrm{N}=31)$ & HS vs COVID19 & HS vs ILD & ILD vs COVID19 \\
\hline Gender, M (\%) & $35(50 \%)$ & $52(62.6 \%)$ & $23(72 \%)$ & $>0.05$ & $<0.05$ & $>0.05$ \\
\hline Age & $58(52-64)$ & $72(58-82)$ & $69(62-75)$ & $<0.001$ & $>0.05$ & $<0.01$ \\
\hline Leukocytes (/ml) & $6.21(5.13-7.43)$ & $6.3(4.68-8.61)$ & $8.77(6.1-11.53)$ & $>0.05$ & $<0.01$ & $<0.01$ \\
\hline Neutrophils $\left(/ \mathrm{mm}^{3}\right)$ & $3.38(2.81-4.22)$ & $4.65(3.32-7.21)$ & $5.78(4.31-8.19)$ & $<0.001$ & $<0.001$ & $>0.05$ \\
\hline Lymphocytes $\left(/ \mathrm{mm}^{3}\right)$ & $2.2(1.71-2.49)$ & $0.92(0.65-1.23)$ & $1.97(0.87-2.2)$ & $<0.001$ & $>0.05$ & $<0.001$ \\
\hline $\mathrm{CRP}(\mathrm{mg} / \mathrm{L})$ & $1.0(0.5-2.4)$ & $60(27-146)$ & $4.8(2.1-9)$ & $<0.001$ & $>0.05$ & $<0.001$ \\
\hline Fibrinogen (g/L) & $2.88(2.56-3.43)$ & $5.24(4.03-6.23)$ & $3.43(3.18-4.15)$ & $<0.001$ & $>0.05$ & $<0.01$ \\
\hline $\mathrm{KL}-6(\mathrm{U} / \mathrm{mL})$ & $254(191-308)$ & $405(277-592)$ & $897(550-1885)$ & $<0.001$ & $<0.001$ & $<0.001$ \\
\hline
\end{tabular}

Data are expressed in median (IQR, inter quartile range)

Data are analyzed with Kruskall Wallis test and post Hoc: DunnTest and with Fisher's test for the sex variable

${ }^{a} \mathrm{HS}$ (healthy subjects): Complete blood count: $\mathrm{N}=62 ; \mathrm{CRP}: \mathrm{N}=52$; Fibrinogen: $\mathrm{N}=46$ 

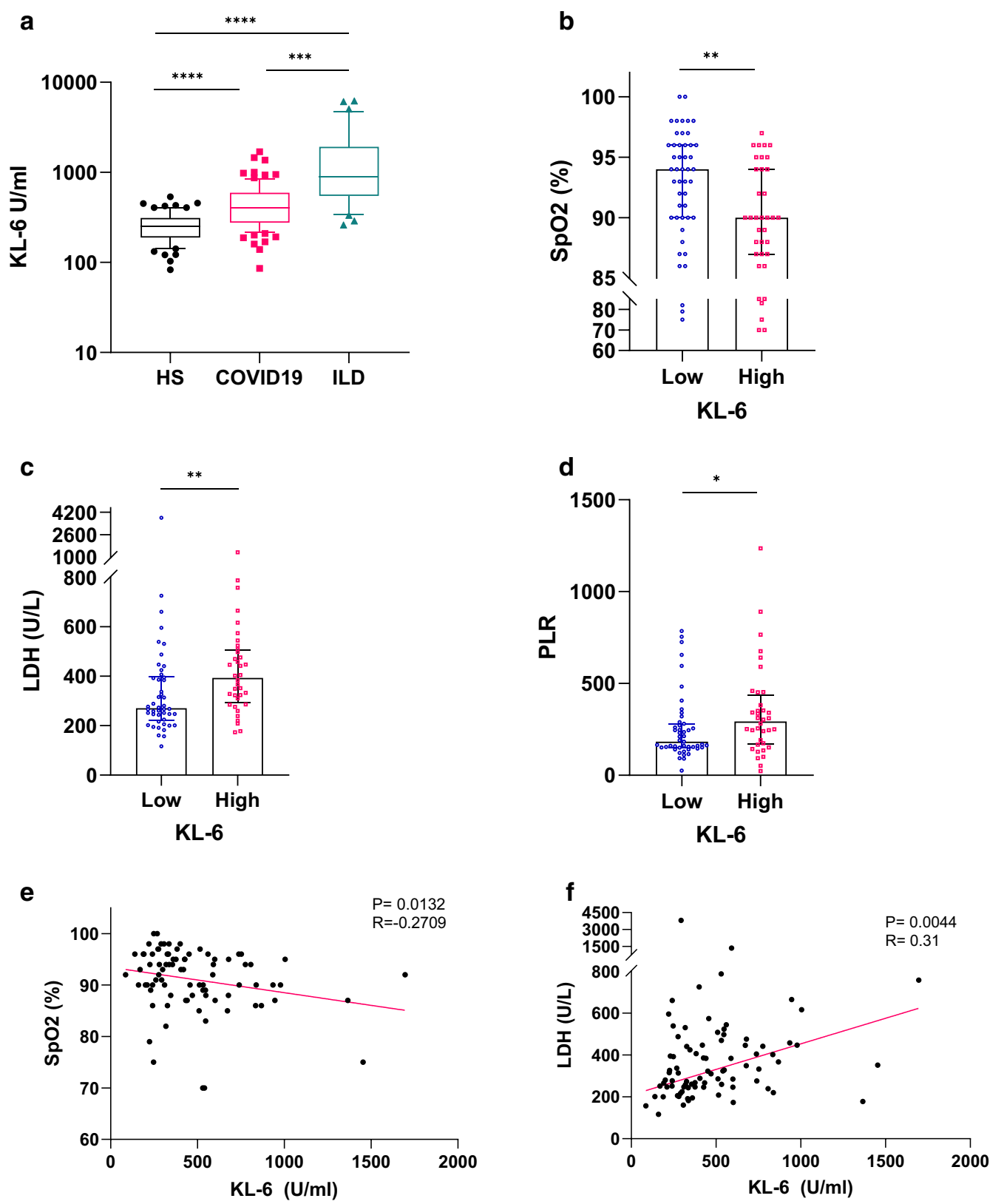

Fig. 1 Multi-panel describing results. a Comparison of KL-6 levels in healthy subjects, COVID-19 subjects, and ILD-subjects. b Comparison of oxygen levels at admission between high and low KL-6 level patients. High KL-6 patients display a significantly lower $\mathrm{SpO}_{2}$. c Comparison of LDH values between high and low KL-6 level patients. High KL-6 patients display a significantly higher LDH value. d Comparison of PLR (platelets/lymphocyte ratio) between high and low KL-6 level patients. High KL-6 patients display a significantly higher PLR. e Correlation between KL- 6 and oxygen levels. f Correlation between KL-6 and LDH levels. Statistics: Data are analyzed by non-parametric unpaired test. a Kruskall Wallis test and with post hoc Dunn-test; b-d. Mann-Whitney test; e, f: Spearman correlation. ${ }^{*} \mathrm{P}<0.05 ;{ }^{* * P}<0.001$; ${ }^{* * *} \mathrm{P}<0.0001$. NB: High KL-6 are KL-6 levels for which values are above mean $+2 S D$

levels $(r=0.31 \mathrm{p}=0.004$, Fig. 1c, $\mathrm{f})$. Concerning platelet/lymphocyte ratio (PLR), we did not find a global correlation with KL-6, but noteworthy, high-KL-6 levels were associated to higher values of PLR $(p=0.04$, Fig. 1d).

\section{Discussion}

KL-6 is known to be linked to alveolar damage [7]. Previous studies have demonstrated that COVID-19 infections were associated to potential lung fibrosing process induced by alveolar damage [8]. Therefore, using 
biomarkers in order to predict lung evolution of severe COVID-19 infections could be of interest in order to identify patients with high risk of experiencing severe lung disease as well as significant parenchymal sequelae.

Our study confirms that KL-6 level could be an indicator of COVID-19 infection severity. This was verified by the parallel impact on oxygen levels. Nevertheless, high KL-6 levels were not associated to more pronounced dyspnea, as this clinical feature was largely encountered in COVID-19 patients, regardless of their KL-6 levels. Similarly, there was no link between high KL-6 levels and ICU admission or death, for which we assume many other factors ought to be considered. Still, high KL-6 levels were interestingly correlated with other indicators of disease severity such as high LDH and PLR, as already mentioned in previous studies $[9,10]$.

Most recent studies focus on the current clinicallaboratory or CT features of COVID-19, but only a few are questioning the long-term impact of such infection. However, tissue inflammation and subsequent healing of the lungs can lead to pulmonary fibrosis. Our study confirms that KL-6-a recognized marker in lung fibrosing process-is increased in COVID-19 patients. This finding is in line with recent studies, describing the rise of other fibrosis biomarkers in this infection [8]. Therefore, the hypothesis of KL-6 as an indicator of lung disease (acute hypoxemia), and even of a possible evolution towards a fibrotic process in the course of a COVID 19 infection must be considered.

\section{Conclusion}

Taken together, these results suggest that KL-6 could be a good biomarker to assess ILD severity in COVID19 infection. Concerning the therapeutic response and prognosis prediction, more studies are necessary.

\section{Abbreviations \\ HS: Healthy subjects; ICU: Intensive care unit; ILD: Interstitial lung disease; KL-6: Krebs von den Lungen 6; LDH: Lactate deshydrogenase; PCR: Polymer- ase chain reaction; PLR: Platelet/lymphocyte ratio; SARS-COV 2: Severe acute respiratory syndrome-coronavirus.}

\section{Acknowledgements}

The authors would like to highlight the work and dedication of the entire COVID-19 clinical investigation team at the University Hospital of Liège: Ancion A., Berg J., Bonhomme O., Bouquegneau A., Bovy C., Bruls S., Darcis G., Defraigne J-O., Ghuysen A., Gilbert A., Heinen V., Lambermont B., Malaise O., Martin M., Nguyen Dang D., Piazza J., Szecel D., Szecel J., Van Cauwenberge H., Von Frenckell C., Vroonen L.

\section{Authors' contributions}

ANF conceptualization, data curation, investigation, writing, visualization, review, editing. LS resources, review. AL resources, review. MH data curation, formal analysis, visualization, review. BD review. FV review. BM review. MM review. RL review. EC conceptualization, methodology, investigation, resources, writing, review. JG conceptualization, methodology, data curation, investigation, writing, review. All authors read and approved the final manuscript.

\section{Funding}

There was no funding for this study. This research did not receive any specific grant from funding agencies in the public, commercial, or not-for-profit sectors.

\section{Availability of data and materials}

The datasets used and/or analysed during the current study are available from the corresponding author on reasonable request.

\section{Ethics approval and consent to participate}

Not applicable.

\section{Consent for publication}

Not applicable.

\section{Competing interests}

The authors declare that they have no competing interests.

\section{Author details}

${ }^{1}$ Department of Respiratory Medicine, CHU Liège, University Hospital of Liège, Domaine Universitaire du Sart-Tilman, B35, 4000 Liège, Belgium. ${ }^{2}$ Department of Clinical Chemistry, CHU Liège, University Hospital of Liège, Domaine Universitaire du Sart-Tilman, B35, 4000 Liège, Belgium. ${ }^{3}$ Intensive Care Unit, CHU Liège, University Hospital of Liège, Domaine Universitaire du Sart-Tilman, B35, 4000 Liège, Belgium. ${ }^{4}$ Department of Infectious Diseases and Immunology, CHU Liège, University Hospital of Liège, Domaine Universitaire du Sart-Tilman, B35, 4000 Liège, Belgium.

Received: 18 May 2020 Accepted: 29 October 2020

Published online: 24 November 2020

\section{References}

1. WHO. Coronavirus disease (COVID-19) Pandemic [Internet]. 2020. https:// www.who.int/emergencies/diseases/novel-coronavirus-2019.

2. CDC. Coronavirus (COVID-19) [Internet]. 2020. https://www.cdc.gov/ coronavirus/2019-ncov/index.html.

3. Venkataraman T, Coleman CM, Frieman MB. Overactive epidermal growth factor receptor signaling leads to increased fibrosis after severe acute respiratory syndrome coronavirus infection. J Virol. 2017;91(12):e00182-17.

4. Guiot J, Moermans C, Henket M, Corhay J-L, Louis R. Blood biomarkers in idiopathic pulmonary fibrosis. Lung. 2017;195(3):273-80.

5. Bonhomme O, André B, Gester F, de Seny D, Moermans C, Struman I, et al. Biomarkers in systemic sclerosis-associated interstitial lung disease: review of the literature. Rheumatology. 2019;58(9):1534-46.

6. Inoue Y, Kaner RJ, Guiot J, Maher TM, Tomassetti S, Moiseev S, et al. Diagnostic and prognostic biomarkers for chronic fibrosing interstitial lung diseases with a progressive phenotype. Chest. 2020;158(2):646-59.

7. Milara J, Ballester B, Montero P, Escriva J, Artigues E, Alós M, et al. MUC1 intracellular bioactivation mediates lung fibrosis. Thorax. 2020;75(2):132-42.

8. Ding M, Zhang Q, Li Q, Wu T, Huang Y. Correlation analysis of the severity and clinical prognosis of 32 cases of patients with COVID-19. Respir Med. 2020;167:105981.

9. Qu R, Ling Y, Zhang Y, Wei L, Chen X, Li X, et al. Platelet-to-lymphocyte ratio is associated with prognosis in patients with Corona Virus Disease-19. J Med Virol. 2020.

10. Han Y, Zhang H, Mu S, Wei W, Jin C, Xue Y, et al. Lactate dehydrogenase, a risk factor of severe COVID-19 patients. Intensive Care Crit Care Med. 2020. https://doi.org/10.1101/2020.03.24.20040162.

\section{Publisher's Note}

Springer Nature remains neutral with regard to jurisdictional claims in published maps and institutional affiliations. 